\title{
Análise de instrumentos de apoio aos profissionais de saúde da atenção primária frente aos casos de violência contra a mulher
}

\author{
Analysis of instruments to support primary care health professionals in cases of violence \\ against women
}

Análisis de instrumentos de apoyo a los profesionales de la salud de atención primaria en casos de violencia contra la mujer

Ianara Fabiana Ramalho Dias Alves ${ }^{1}$, Gilberto José Montaño Góes de Mendonça ${ }^{1}$, Domennica Gomes Pecorelli ${ }^{1}$, Márcya Cândida Casimiro de Oliveira ${ }^{1}$, Nathália de Oliveira Azevedo ${ }^{1}$, André Luís Belmiro Moreira Ramos ${ }^{1}$, Amanda Apolori Tissiani ${ }^{1}$, Layza de Souza Chaves Deininger ${ }^{1 *}$.

\section{RESUMO}

Objetivo: Analisar a literatura científica sobre instrumentos de apoio aos profissionais de saúde da atenção primária frente aos casos de violência contra a mulher. Métodos: Trata-se de uma revisão integrativa realizada em março e maio de 2021, nas plataformas MEDLINE, SciELO e LILACS, utilizando descritores controlados. Foram selecionados artigos publicados nos últimos cinco anos, nos idiomas português, inglês e espanhol, excluindo-se monografias dissertações, teses, artigos duplicados e artigos que não guardavam correlação com o objeto do estudo. Resultados: Dentre os 17 artigos selecionados para compor o corpo amostral, predominou o tema relativo as estratégias de intervenções que os profissionais de saúde utilizaram frente às situações de violência contra a mulher com nove artigos, seguido das dificuldades e possibilidades de atuação dos profissionais da atenção primária à saúde na rede com quatro estudos, e educação em saúde e políticas públicas relacionadas com apenas um em cada. Considerações finais: A assistência ofertada pelos profissionais de saúde não utiliza um instrumento específico, necessita de reorganização do processo de trabalho na atenção primária com ações intersetoriais formando uma rede de atenção voltada às mulheres vítimas de violência a fim de garantir a integralidade do cuidado a estas vítimas.

Palavras-chave: Atenção primária à saúde, Documentação, Protocolos, Registros, Violência contra a mulher.

\begin{abstract}
Objective: To analyze the scientific literature on instruments to support primary care health professionals in cases of violence against women. Methods: This is an integrative review carried out in March and May 2021, on MEDLINE, SciELO and LILACS platforms, using controlled descriptors. Articles published in the last five years, in Portuguese, English and Spanish were selected, excluding monographs, dissertations, theses, duplicate articles and articles that did not correlate with the object of study. Results: Among the 17 articles selected to compose the sample body, the theme related to intervention strategies that health professionals used in situations of violence against women predominated with nine articles, followed by the difficulties and possibilities of action of primary care professionals to network health with four studies, and health education and related public policies with only one in each. Final considerations: The assistance offered by health professionals does not use a specific instrument, it needs to reorganize the work process in primary care with intersectoral actions, forming a care network aimed at women victims of violence in order to ensure comprehensive care for these victims.
\end{abstract}

Keywords: Primary health care, Documentation, Protocols, Records, Violence against women.

${ }^{1}$ Faculdade de Ciências Médicas da Paraíba (FCM-PB), João Pessoa - PB.

*E-mail: layzadeininger@gmail.com

SUBMETIDO EM: 6/2021

ACEITO EM: 6/2021

PUBLICADO EM: 6/2021 


\section{RESUMEN}

Objetivo: Analizar la literatura científica sobre instrumentos de apoyo a los profesionales de la salud de atención primaria en casos de violencia contra la mujer. Métodos: Se trata de una revisión integradora realizada en marzo y mayo de 2021, en plataformas MEDLINE, SciELO y LILACS, utilizando descriptores controlados. Se seleccionaron artículos publicados en los últimos cinco años, en portugués, inglés y español, excluyendo monografías, disertaciones, tesis, artículos duplicados y artículos que no tuvieran correlación con el objeto de estudio. Resultados: Entre los 17 artículos seleccionados para componer el cuerpo muestral, predominó la temática relacionada con las estrategias de intervención que los profesionales de la salud utilizaron en situaciones de violencia contra la mujer con nueve artículos, seguido de las dificultades y posibilidades de acción de los profesionales de atención primaria para la salud en red con cuatro estudios, y educación para la salud y políticas públicas relacionadas con solo una en cada una. Consideraciones finales: La atención brindada por los profesionales de la salud no utiliza un instrumento específico, es necesario reorganizar el proceso de trabajo en la atención primaria con acciones intersectoriales, conformando una red de atención dirigida a las mujeres víctimas de violencia con el fin de asegurar una atención integral a estas víctimas.

Palabras clave: Primeros auxilios, Documentación, Protocolos, Registros, La violencia contra las mujeres.

\section{INTRODUÇÃO}

Segundo a Organização Mundial da Saúde (OMS), a violência é o ato de exercer força ou poder contra qualquer indivíduo, esteja ele isolado ou em grupo, sendo atualmente reconhecida como um problema de saúde pública devido às repercussões negativas na qualidade de vida da vítima, além de sua elevada prevalência no cenário mundial (SANTOS MS, et al., 2021; ESPERANDIO EG, et al., 2020).

A violência pode ser subdividida em grupos específicos na sociedade, a exemplo, das mulheres. Nesse contexto, apesar do termo violência contra mulher ter sido talhado há cerca de 20 anos pelo movimento feminista, apenas se difundiu com a compreensão de que esse crime se desenvolve sobre diferentes formas, como a física, psicológica, sexual, patrimonial e moral, levadas a efeito através de atos omissivos ou comissivos que infiram danos a essas esferas da mulher (SACRAMENTO LT e REZENDE MM, 2006; SANTOS SC, et al., 2018; OLIVEIRA GL, et al., 2020).

$\mathrm{Na}$ esfera global, a OMS estima que uma a cada três mulheres já foram vítimas de violência doméstica (VD), razão pela qual a referida entidade já considera a VD uma epidemia mundial, destacando sua maior prevalência em países de média e baixa renda, dado este que inclui o Brasil, com uma média de uma mulher assassinada a cada duas horas, o que o coloca em uma posição elevada no ranking de países com pior índice de violência contra a mulher (SANTOS SC, et al., 2018; BHONA FM, 2019).

Destaca-se que esses dados persistem apesar de o combate à violência contra a mulher ser uma questão bastante debatida, ganhando especial relevo em razão da variabilidade e intensidade dos danos causados às vítimas (MENDONÇA CF, et al., 2020).

O enquadramento da violência contra mulher como problema de saúde pública se deve também aos danos que recaem sobre a saúde física e psicológica da mulher, levando a quadros patológicos graves a exemplo da depressão, trauma, dificuldade de relacionar-se com outras pessoas, além dos danos físicos e reprodutivos (SIGNORELLI MC, et al., 2018; OLIVEIRA GL, et al., 2020).

As circunstâncias se agravam com a fragilidade do atendimento prestado pelo Sistema Único de Saúde (SUS), nessa perspectiva, é fator de revitimização a impunidade de seus agressores, a invisibilidade da violência e inexistência de uma rede articulada de apoio, o que culmina para o aumento da sensação de impotência e vulnerabilidade social das vítimas. Diante do exposto, a atuação do profissional de saúde deve ser humanizada, para, através do acolhimento e escuta qualificada, seja possível reconhecer os sinais e sintomas da violência, fatos que nem sempre são revelados claramente. Assim, o atendimento não deve se limitar ao atendimento das dores e feridas físicas, é preciso encaminhar a usuária pela rede de apoio, buscando a integralidade do cuidado (SIGNORELLI MC, et al., 2018; OLIVEIRA GL, et al., 2020). 
Vale salientar que os profissionais da saúde se tornaram uma das principais ferramentas de identificação das vítimas de violência doméstica, sobretudo os que atuam na Atenção Primária à Saúde (APS), posto que, na qualidade de principal porta de entrada dos serviços do SUS, é quem geralmente atua no primeiro atendimento das mulheres, como também podem desenvolver ações de prevenção de agravos e promoção à saúde (AMARIJO CL, et al., 2018; CAMPOS IO, et al., 2020)

Entretanto, o desempenho desse papel central é ofuscado por obstáculos, que vão desde a formação inicial e continuada até a desarticulação da intersetorialidade dos órgãos e entidades de apoio e proteção à mulher, criando uma instabilidade do fluxo de atendimento, e incluindo a dificuldade de identificação, que leva a subnotificação dos casos, o que justifica a necessidade de novos estudos acerca da temática (CAMPOS IO, et al., 2020; D'OLIVEIRA AFPL, et al., 2020).

Desse modo, o presente estudo objetivou analisar instrumentos de apoio aos profissionais de saúde da atenção primária frente aos casos de violência contra a mulher.

\section{MÉTODOS}

Trata-se de uma revisão integrativa da literatura, a qual procurou reunir e organizar estudos com diferentes métodos, com o intuito de combinar os dados teóricos e empíricos favorecendo uma melhor compreensão do objeto de estudo (MENDES KDS, et al., 2008).

As etapas recomendadas para uma revisão abrangente são as seguintes: estabelecimento do problema; após, determinar os critérios de inclusão e exclusão, selecione a amostra final; em seguida, informações coletadas dos estudos pesquisados; na sequência, análise de resultados (identificar semelhanças e conflitos); por fim, discussão dos resultados da pesquisa (POMPEO DA, et al., 2009). O estudo apresentou a seguinte questão norteadora: Há instrumentos de apoio aos profissionais de saúde da atenção primária frente aos casos de violência contra a mulher?

A composição da amostra foi realizada por meio de buscas nas seguintes bases de dados: Revista Literatura Latino-americana e do Caribe em Ciências da Saúde (LILACS), Scientific Electronic Library Online (SciELO) e Sistema Online de Busca e Análise de Literatura Médica (MEDLINE). As buscas foram direcionadas por descritores controlados combinados com operadores booleanos: violência contra a mulher AND atenção primária à saúde AND documentação OR registros OR protocolos e domestic violence AND women AND primary health care AND protocols.

Os critérios de inclusão utilizados foram periódicos disponíveis na íntegra nos idiomas português, inglês e espanhol, publicados no período entre 2017 e 2021. Já os critérios de exclusão foram: monografias, dissertações, teses, artigos duplicados ou que não incluíssem o objetivo do presente estudo. As buscas foram realizadas no período de março a maio de 2021. Inicialmente foram encontradas 66 publicações (Figura 1).

Figura 1 - Fluxograma de composição da amostra.

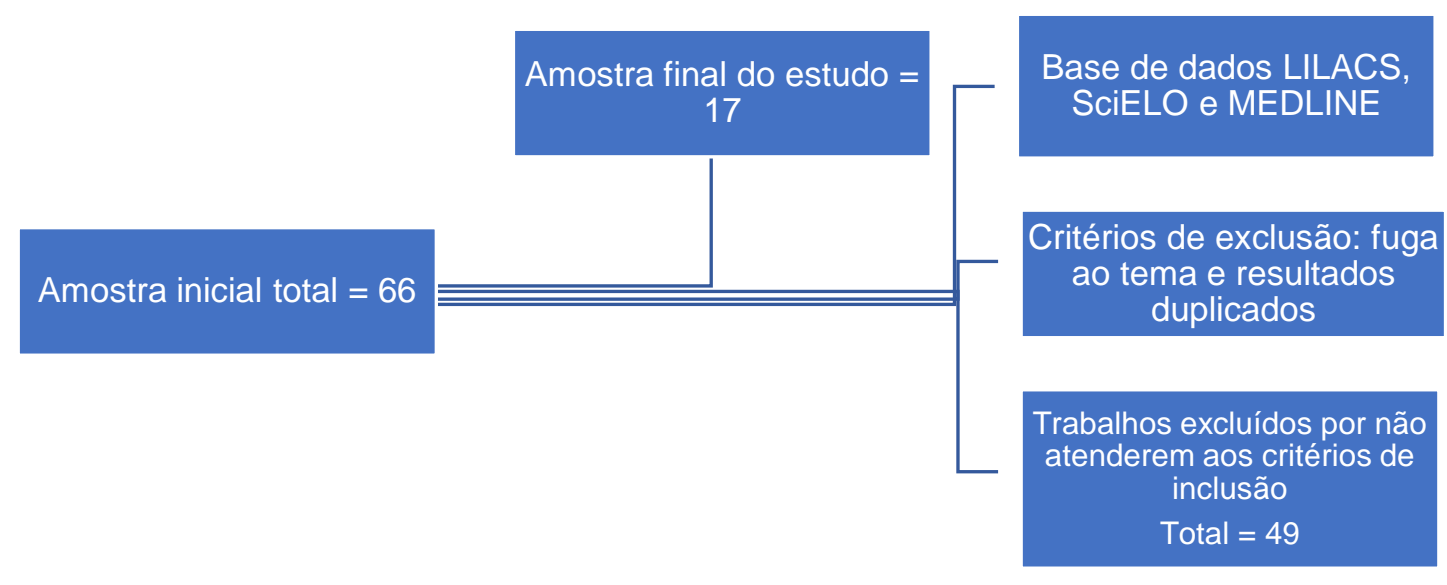

Fonte: Alves IFRD, et al., 2021. 
Foi realizada uma leitura inicial do título, resumo e descritores para seleção daqueles que se adequavam ao objeto de estudo. Quando não se conseguiu averiguar essa adequação nessa etapa, o estudo foi lido na íntegra para verificar se atendia aos objetivos desta pesquisa. Posteriormente, todos os artigos selecionados foram lidos na íntegra para fazer uma análise minuciosa e apresentar uma sumarização desses estudos.

A seleção dos artigos foi realizada por meio de duas etapas: na primeira, foram utilizados os filtros presentes nas bases de dados consultadas, norteados pelos critérios de inclusão: data de publicação; idioma de publicação; documentos disponíveis. Em seguida, os artigos selecionados nesse momento foram recuperados e submetidos a uma nova seleção, norteada pelos critérios de inclusão. Já na segunda etapa, foram excluídos os artigos duplicados entre as bases de dados consultadas. A análise quantitativa das publicações incluídas nesta revisão levou em consideração a área de conhecimento da qual o artigo estava relacionado e o quantitativo de artigos por ano de publicação. O procedimento de análise qualitativa dos dados foi realizado com uma leitura inicial destes resumos, a fim de se classificar tais artigos quanto ao tema estudado ou tipo de estudo.

Para facilitar a inspeção dos resultados foi construído um quadro com os seguintes elementos: autores, título, periódico, ano de publicação, código em cada artigo selecionado numerado em ordem decrescente por ano de publicação (Violência Contra a Mulher - VCM) e principais resultados, apresentando descritivamente a porcentagem de artigos revisados versus ano de publicação, áreas de revistas e por categorias (Quadro 1).

\section{RESULTADOS}

Na sequência dessa investigação, foram excluídos 49 estudos no total, após leitura dos resumos, visto que estavam duplicados entre as bases de dados utilizadas. Outros estudos foram excluídos por falta de adequação aos critérios estabelecidos (apresentavam tema principal diverso do objeto de pesquisa, tais como: fatores socioeconômicos, clínicos, experiência de violência, autopercepção da saúde). Desses estudos, 17 compuseram a amostra final.

No que diz respeito ao período de publicação, percebeu-se uma distribuição nos últimos 4 anos, com maior número de artigos publicados em $2020 \mathrm{com}$ um total de $41,17 \%$ dos artigos, mostrando uma preocupação maior dos pesquisadores no ano em que se iniciou a pandemia da COVID-19. O ano de 2019 apresentou $11,76 \%$ dos documentos; $23,52 \%$ dos textos foram publicados em $2018 ; 23,52 \%$ dos artigos foram publicados em 2017. 
Quadro 1 - Informações das publicações analisadas, segundo ordem decrescente por ano de publicação. Violência contra a mulher (VCM).

\section{Autores}

\begin{tabular}{|c|c|c|c|c|}
\hline Autores & Título & Periódico & Ano/Código & Principais resultados \\
\hline $\begin{array}{l}\text { BEARZI PSS, et } \\
\text { al. }\end{array}$ & Trilhas para o enfrentamento da violência contra a mulher & Estudos Feministas & $\begin{array}{c}2020 \\
\text { (VCM1) }\end{array}$ & $\begin{array}{l}\text { Apresentam condutas para a reflexão sobre as potencialidades da } \\
\text { Atenção Básica. }\end{array}$ \\
\hline $\begin{array}{l}\text { ESPERANDIO } \\
\text { EG, et al. }\end{array}$ & $\begin{array}{l}\text { Violência íntima: experiências de mulheres na Atenção } \\
\text { Primária à Saúde no Rio de Janeiro, RJ, Brasil }\end{array}$ & $\begin{array}{l}\text { Interface (Botucatu, } \\
\text { Online) }\end{array}$ & $\begin{array}{c}2020 \\
(\mathrm{VCM} 2)\end{array}$ & $\begin{array}{l}\text { Mostraram dificuldades de revelação da violência íntima e } \\
\text { possibilidades de atuação da atenção primária à saúde. }\end{array}$ \\
\hline $\begin{array}{l}\text { MAGALHÃES } \\
\text { VMPR, et al. }\end{array}$ & $\begin{array}{l}\text { Validação de álbum seriado para enfermeiros da atenção } \\
\text { básica sobre violência doméstica contra a mulher }\end{array}$ & Cogitare enfermagem & $\begin{array}{c}2020 \\
(\mathrm{VCM})\end{array}$ & $\begin{array}{l}\text { Validação de álbum como tecnologia educativa com ações de } \\
\text { educação em saúde realizadas por enfermeiros. }\end{array}$ \\
\hline $\begin{array}{l}\text { SILVA VG e } \\
\text { RIBEIRO PM }\end{array}$ & $\begin{array}{l}\text { Violência contra as mulheres na prática de enfermeiras da } \\
\text { atenção primária à saúde }\end{array}$ & $\begin{array}{l}\text { Escola Anna Nery Revista } \\
\text { de Enfermagem }\end{array}$ & $\begin{array}{c}2020 \\
(\mathrm{VCM} 4)\end{array}$ & $\begin{array}{l}\text { Assistência de enfermagem às mulheres que sofrem violência e } \\
\text { capacitação para o reconhecimento da violência. }\end{array}$ \\
\hline $\begin{array}{l}\text { MOTA JA e } \\
\text { AGUIAR RS }\end{array}$ & $\begin{array}{l}\text { Percepções de enfermeiros da atenção primária no } \\
\text { atendimento às mulheres vítimas de violência sexual }\end{array}$ & Nursing & $\begin{array}{l}2020 \\
(\text { VCM5) }\end{array}$ & $\begin{array}{l}\text { A falta de conhecimento sobre e a dificuldade na identificação da } \\
\text { violência sexual podem resultar na subnotificação. }\end{array}$ \\
\hline $\begin{array}{l}\text { MENDONÇA } \\
\text { CF, et al. }\end{array}$ & $\begin{array}{l}\text { Violência na Atenção Primária em Saúde no Brasil: uma } \\
\text { revisão integrativa da literatura }\end{array}$ & Ciência \& Saúde Coletiva & $\begin{array}{c}2020 \\
(\mathrm{VCM})\end{array}$ & $\begin{array}{l}\text { A invisibilidade da violência na atenção primária do Brasil e a } \\
\text { necessidade de reorganização do processo de trabalho. }\end{array}$ \\
\hline $\begin{array}{l}\text { OLIVEIRA GL, } \\
\text { et al. }\end{array}$ & $\begin{array}{l}\text { Obstáculos e facilitadores para o cuidado de mulheres em } \\
\text { situação de violência doméstica na atenção primária em } \\
\text { saúde: uma revisão sistemática }\end{array}$ & $\begin{array}{l}\text { Interface (Botucatu, } \\
\text { Online) }\end{array}$ & $\begin{array}{c}2020 \\
(\text { VCM7) }\end{array}$ & $\begin{array}{l}\text { Obstáculos e facilitadores para o cuidado de mulheres em } \\
\text { situação de violência doméstica na atenção primária à saúde no } \\
\text { Brasil. }\end{array}$ \\
\hline $\begin{array}{l}\text { SEHNEM GD, et } \\
\text { al. }\end{array}$ & $\begin{array}{l}\text { Violência contra as mulheres: atuação da enfermeira na } \\
\text { atenção primária à saúde }\end{array}$ & $\begin{array}{l}\text { Revista de enfermagem da } \\
\text { UFSM }\end{array}$ & $\begin{array}{c}2019 \\
(\text { VCM8) }\end{array}$ & $\begin{array}{l}\text { A falta de abordagem do tema na formação acadêmica e } \\
\text { profissional e a desarticulação da rede de atenção. }\end{array}$ \\
\hline $\begin{array}{l}\text { NASCIMENTO } \\
\text { VF, et al. }\end{array}$ & $\begin{array}{l}\text { Desafios no atendimento aos casos de violência doméstica } \\
\text { contra a mulher em um município Matogrossense }\end{array}$ & $\begin{array}{l}\text { Arquivos de ciências da } \\
\text { saúde da UNIPAR }\end{array}$ & $\begin{array}{c}2019 \\
(\text { VCM9) }\end{array}$ & $\begin{array}{l}\text { Obstáculos encontrados pelos profissionais na identificação e } \\
\text { enfrentamento diante de denúncias e acompanhamento dos } \\
\text { envolvidos. }\end{array}$ \\
\hline $\begin{array}{l}\text { AMARIJO CL, et } \\
\text { al. }\end{array}$ & $\begin{array}{l}\text { Assimilação teórica e prática da violência doméstica: } \\
\text { profissionais de enfermagem atendendo vítimas na } \\
\text { atenção primária }\end{array}$ & $\begin{array}{l}\text { Revista de enfermagem da } \\
\text { UERJ }\end{array}$ & $\begin{array}{c}2018 \\
(\text { VCM10) }\end{array}$ & $\begin{array}{l}\text { Os componentes da violência doméstica contra a mulher (VDCM): } \\
\text { conceito, imagem, atitude e detecção dos casos. }\end{array}$ \\
\hline $\begin{array}{l}\text { SANTOS, SC, et } \\
\text { al. }\end{array}$ & $\begin{array}{l}\text { Violência contra a mulher: como os profissionais na } \\
\text { atenção primária à saúde estão enfrentando esta } \\
\text { realidade? }\end{array}$ & Saúde e pesquisa & $\begin{array}{c}2018 \\
(\mathrm{VCM} 11)\end{array}$ & $\begin{array}{l}\text { Identifica as formas de assistência prestada pelos profissionais da } \\
\text { atenção primária à mulher vítima de violência no município de } \\
\text { Buíque }(P E) \text {. }\end{array}$ \\
\hline ARBOIT J, et al. & $\begin{array}{l}\text { Violência doméstica contra mulheres rurais: práticas de } \\
\text { cuidado desenvolvidas por agentes comunitários de saúde }\end{array}$ & Saúde e Sociedade & $\begin{array}{c}2018 \\
(\mathrm{VCM} 12)\end{array}$ & $\begin{array}{l}\text { As agentes comunitárias de saúde encontravam possibilidades de } \\
\text { identificar e intervir nas situações de VCM rurais. }\end{array}$ \\
\hline $\begin{array}{l}\text { SIGNORELLI } \\
\text { MC, et al. }\end{array}$ & $\begin{array}{l}\text { Violência doméstica contra mulheres, políticas públicas e } \\
\text { agentes comunitários de saúde na Atenção Primária } \\
\text { Brasileira }\end{array}$ & Ciência \& Saúde Coletiva & $\begin{array}{c}2018 \\
(\mathrm{VCM} 13)\end{array}$ & $\begin{array}{l}\text { Existe um hiato entre políticas públicas direcionadas à VCM a } \\
\text { nível federal e sua aplicação a nível local e o papel chave de } \\
\text { agentes comunitários de saúde. }\end{array}$ \\
\hline $\begin{array}{l}\text { SILVA NNF, et } \\
\text { al. }\end{array}$ & $\begin{array}{l}\text { Atuação dos enfermeiros da atenção básica a mulheres } \\
\text { em situação de violência }\end{array}$ & Enfermagem em foco & $\begin{array}{c}2017 \\
(\mathrm{VCM} 14)\end{array}$ & $\begin{array}{l}\text { O tema requer dos enfermeiros maior interação e conhecimento } \\
\text { sobre o assunto. }\end{array}$ \\
\hline $\begin{array}{l}\text { NETTO LA, et } \\
\text { al. }\end{array}$ & $\begin{array}{l}\text { As redes sociais de apoio às mulheres em situação de } \\
\text { violência pelo parceiro íntimo }\end{array}$ & $\begin{array}{l}\text { Texto \& contexto } \\
\text { enfermagem }\end{array}$ & $\begin{array}{c}2017 \\
(\mathrm{VCM} 15)\end{array}$ & $\begin{array}{l}\text { A rede de apoio nos serviços obtidos no hospital, posto de saúde, } \\
\text { centro especializado de atendimento e delegacia. }\end{array}$ \\
\hline ARBOIT J, et al. & $\begin{array}{l}\text { Atenção à saúde de mulheres em situação de violência: } \\
\text { desarticulação dos profissionais em rede }\end{array}$ & $\begin{array}{l}\text { Escola de Enfermagem da } \\
\text { USP }\end{array}$ & $\begin{array}{c}2017 \\
(\mathrm{VCM} 16)\end{array}$ & $\begin{array}{l}\text { Os profissionais de saúde tiveram suas ações limitadas pela } \\
\text { ausência de integração entre os profissionais e serviços da rede. }\end{array}$ \\
\hline $\begin{array}{l}\text { MACHADO } \\
\text { MES, et al. }\end{array}$ & $\begin{array}{l}\text { Percepção de profissionais de saúde sobre violência } \\
\text { contra a mulher: estudo descritivo }\end{array}$ & $\begin{array}{l}\text { Online Brazilian Journal } \\
\text { Nursing }\end{array}$ & $\begin{array}{c}2017 \\
(\mathrm{VCM} 17)\end{array}$ & $\begin{array}{l}\text { (Des) conhecimento sobre notificação e denúncia; (Des) } \\
\text { conhecimento sobre a lei que pune quem comete VCM. }\end{array}$ \\
\hline
\end{tabular}

Fonte: Alves IFRD, et. al., 2021. 
Dos 17 estudos analisados sobre a violência contra a mulher (VCM) neste estudo, 52,94\% (VCM3, VCM4, VCM5, VCM8, VCM10, VCM14, VCM15, VCM16, VCM17) estavam vinculados a revistas da área da Enfermagem, 17,64\% dos textos (VCM6, VCM12, VCM13) à área da Saúde Coletiva, $11,76 \%$ dos artigos (VCM2, VCM7) à área da Medicina, 11,76\% das pesquisas (VCM9, VCM11) evidenciaram uma revista de Ciência Biomédicas e da Saúde que envolve todas as áreas e a área das Ciências Sociais (questões de gênero) foram encontradas em apenas $5,88 \%$ dos estudos (VCM1).

A partir da análise dos 17 artigos selecionados nesta pesquisa, foi realizada uma classificação quanto ao tema estudado ou tipo de estudo, em que foram identificadas as seguintes categorias e sua incidência dentre os textos selecionados.

$\mathrm{Na}$ categoria estratégias $(52,94 \%)$, foram incluídos os estudos (VCM1, VCM4, VCM5, VCM9, VCM10, VCM11, VCM12, VCM14, VCM17) em que estavam descritas capacitações, estratégias e/ou técnicas de intervenções que os profissionais de saúde da Atenção Básica utilizaram frente às situações de violência contra a mulher.

Na sequência, a categoria dificuldades e possibilidades (23,52\%) incluiu os artigos (VCM2, VCM8, VCM15, VCM16) que mostravam as dificuldades de revelação da violência íntima pelas vítimas e as possibilidades de atuação dos profissionais da atenção primária à saúde.

Na categoria revisão de literatura (11.76\%), foram incluídos os textos (VCM6, VCM7) que mencionavam a realização de uma revisão da literatura sobre o tema da VCM, seja de forma geral ou específica.

Em menor porcentagem, a categoria atividades de educação em saúde (5,88\%) agregou o artigo (VCM3) com vistas à promoção de informações sobre a violência doméstica contra a mulher, favorecendo o processo de comunicação entre as mulheres e os profissionais de saúde e a categoria avaliação/análise de políticas públicas $(5,88 \%)$ foi inserido o texto (VCM13) que avaliava uma ou mais política pública, legislação de proteção à mulher e os atendimentos de saúde.

\section{DISCUSSÃO}

A realidade da violência contra a mulher não conhece fronteiras, caracterizando um problema mundial, a ponto de a Organização das Nações Unidas (ONU) editar sua Declaração pela Eliminação da Violência Contra as Mulheres (DEVAM), em 1993, reconhecendo como forma de discriminação e violação de direitos humanos a violência contra a mulher (AMARIJO CL, et al., 2018).

O Brasil, em 1994, na cidade de Belém do Pará, sediou a Assembleia Geral da Comissão Interamericana de Direitos Humanos (CIDH) onde se pactuou a Convenção Interamericana para Prevenir, Punir e Erradicar a Violência contra a Mulher - conhecida como Convenção de Belém do Pará - a partir da qual qualquer ato baseado no gênero, que gerasse dano às mulheres, passou a ser entendida como violência contra mulher. Entretanto, apenas com a edição da Lei n. 11.340/2006 - "Lei Maria da Penha" - foi estabelecido um marco legal na proteção contra a mulher no país, prevendo medidas multidisciplinar às mulheres, possibilitando a integração de profissionais da área de saúde, psicologia e Direito na elaboração de ações articuladas em rede para o cuidado integral da mulher (NETTO LA, et al., 2017).

A propósito, as medidas protetivas e inibitórias de ilícitos penais foram ampliadas com o advento da Lei $13.104 / 2015$, ao prever o feminicídio como circunstância qualificada do crime de homicídio. Todavia, a edição de disposições legais não é suficiente para extirpar a VCM do seio da sociedade, sendo necessária uma estreita comunicação entre as políticas públicas e as legislações para o enfrentamento dessa epidemia, sob pena de continuarmos amargurando números como os da OMS segundo os quais $30 \%$ das mulheres já foram vítimas de violência física e/ou sexual (ESPERANDIO EG, et al., 2020; SEHNEM GD, et al., 2019).

Além disso, destaca-se que, no Brasil, dados do Sistema de Informação de Agravos de Notificação (SINAN) demonstram uma escalada no aumento da violência de gênero, colocando-o na sétima posição de um ranking mundial, sendo cometidos aproximadamente 13 feminicídios por dia. Fato que pode ser ainda pior devido a subnotificação desse sistema de informação (SILVA VG e RIBEIRO PM, 2020). 
Sendo a VCM um problema de saúde pública, a APS, enquanto porta de entrada do SUS, deve assumir seu protagonismo no cuidado das mulheres sujeitas à violência, sobretudo ante seus princípios norteadores de integralidade, resolutividade, humanização, continuidade do cuidado e territorialização que a coloca em posição estratégica na prevenção de agravos, promoção da saúde e coordenação do cuidado às vítimas de violência, estando apta a encadear diferentes componentes para compor a integralidade da assistência necessária (ARBOIT J, et al., 2017; MACHADO MES, et al, 2017; MENDONÇA CF, et al., 2020).

Outro fator relevante do protagonismo da APS frente a essa problemática é a possibilidade da utilização de tecnologia leve como 0 acolhimento, escuta qualificada e construção do vínculo que favorecem 0 estabelecimento de uma relação de confiança e, por vezes, de afeto entre os profissionais e as usuárias, 0 que facilitaria a comunicação franca e aberta entre aqueles atores (SILVA VG e RIBEIRO PM, 2020).

Contudo, diversas causas multifatoriais impedem esse protagonismo, iniciando pela dificuldade de constatar os sinais de violência, tendo em vista que alguns apresentam caráter subjetivo, acrescentando ainda o mascaramento de alguns pelas próprias vítimas. Isto porque a violência nas relações conjugais se manifesta não apenas fisicamente, mas também na relação íntima do casal, causando, através da comunicação violenta, agravos morais e psicológicos, os quais são ainda mais difíceis de identificar nesse contexto múltiplo de violência (D'OLIVEIRA AFPL, et al., 2020).

Assim, é necessário destacar que alguns sinais e sintomas são comumente encontrados em mulheres sujeitas a violência, devendo os profissionais de saúde estarem atentos para identificar situações, ainda que omitidas pela usuária, a saber: depressão, ansiedade e distúrbios psíquicos e do sono, comportamento e pensamento suicida, cefaleias, tonturas, distúrbios gastrointestinais, picos hipertensivos, dores crônicas sem explicação aparentes, lesões físicas sem explicação condizente com o relato, além do eventual abuso de álcool e/ou drogas ilícitas (MACHADO MES, et al., 2017; SILVA NNF, et al., 2017).

No contexto conjugal, os relatos noticiam violência sexual, através de assédio sexual, manutenção de relação sexual não consentida e violência psicológica, com a humilhação e outros mecanismos que levam a diminuição da autoestima potencialmente descambando para uma violência moral, quando presente calúnia, injúria ou difamação, além de violência patrimonial, onde o agressor promove a dependência econômica da vítima através de retenção ou destruição de bens e objetos da vítimas (BEARZI PSS, et al., 2020).

O mascaramento da realidade de violência pela vítima e o receio em procurar as variadas formas de cuidado deve-se ao medo do agressor, que em sua maioria, são pessoas com quem mantém relação íntima e que sujeita as vítimas a constantes ameaças de morte própria ou de parentes, relatando-se também dependência de econômica, vergonha pelos julgamentos que podem vir até mesmo de pessoas próximas, culpando-a pela violência, crença nas promessas de mudança do agressor, receio de perder os filhos, dentre outros (AMARIJO CL, et al., 2018).

Ainda a despeito da subjetividade das vítimas, sabe-se que os constantes ataques sofridos pelas mulheres nesse contexto de violência afetam negativamente suas relações sociais, que, em decorrência da instabilidade emocional que nela se instaura, reduz significativamente sua rede de amizades, a qual poderia prestar solidariedade e apoio material e emocional, tendo, por vezes, que buscarem sozinhas ajudas aos órgãos de apoio (NETTO LA, et al., 2017).

Ante toda essa conjuntura, percebe-se a necessidade de criar formas de identificar, até de maneira precoce, estes agravos, a fim de os prevenir, estando o agente comunitário de saúde (ACS), apto a assumir papel de destaque no processo do cuidado, devido ao seu maior vínculo e interação com a comunidade, por nela habitar (NASCIMENTO VF, et al., 2019).

Outro ponto relatado pelos profissionais de saúde tem sido a formação profissional na abordagem da violência doméstica, que se inicia ainda nas universidades e prorroga-se para a vida laboral, com a falta de treinamentos e capacitação, que geram desconhecimento tanto de questões legais, quanto de mecanismos para abordar o tema, para realizar um atendimento sistêmico às vítimas de VCM (MENDONÇA CF, et al. 2020). 
Logo, é oportuno dizer que a escassez de conhecimento sobre o assunto aliado a uma escuta e acolhimento frágeis advém da falta de empatia e ignorância sobre os efeitos negativos da violência na vida das mulheres, conduzindo o profissional a um manejo apenas biomédico, relegando a integralidade do cuidado. Para fazer frente a essa formação deficiente, muito se sugere uma estratégia de educação continuada e incremento desses temas desde a graduação (MOTA JA e AGUIAR RS, 2020; SILVA VG e RIBEIRO PM, 2020).

Também vale destacar a relevância da desarticulação ou inexistência de uma rede intersetorial como obstáculo à integralidade do cuidado às vítimas de violência de gênero. Para os profissionais de saúde, referida desarticulação impede-os de reconhecer os serviços e instrumentos sociais para os quais poderia encaminhar visando atender a todas as necessidades de cuidado das vítimas, apesar de haver no uma vasta rede de serviços destinados ao acolhimento e proteção dos direitos das mulheres expostas à violência (D'OLIVEIRA AFPL, et al. 2020; AIRBOT J, et al., 2018; NASCIMENTO VF, et al., 2019).

Nesse sentido, a APS é a principal porta de entrada do usuário no serviço de saúde e, sendo a violência uma realidade, o profissional de saúde deve estar habilitado a atender às usuárias que a eles recorrem. Contudo, foi identificado que não há uma sistematização dos procedimentos adotados, que aliado à falta de conhecimento e até empatia, deixa de prover o cuidado integral que a paciente necessita, o que não deve ser justificativa para que atitudes positivas não sejam tomadas isoladamente ou em equipe (D'OLIVEIRA AFPL, et. al., 2020; SILVA NNF, et al., 2017; BEARZI PSS, et al., 2020; MOTA JA e AGUIAR RS, 2020).

Assim, os profissionais de saúde devem respeitar a individualidade, privacidade e intimidade, preocupando-se que $o$ acolhimento e a escuta dessas usuárias sejam realizada em locais apropriados e reservados, garantindo o sigilo das informações que forem fornecidas; permitir que a usuária se expresse adequadamente, sem interrupções e sem buscar detalhes que não sejam relevantes para o caso; orientar e fornecer suporte, utilizando uma linguagem acessível à população; estimular, na perspectiva do autocuidado, que a usuária tome iniciativas, a fim de proteger a si mesma e interromper o ciclo de violência; orientar e coordenar o cuidado ao longo de toda rede de atenção, bem como nos diversos instrumentos e organizações sociais destinadas ao cuidado da vítima (MACHADO MES, et al., 2017; NASCIMENTO VF, et al., 2019; ESPERANDIO EG, et al., 2020)

Para tanto, o profissional deve conhecer todos os órgãos que podem auxiliar as mulheres além da Delegacia Especializada da Mulher, afinal não se trata apenas de uma questão policial. É exatamente essa falta de articulação e responsabilização, aliados à burocracia e ao processo de revitimização, já que são obrigadas a reviver toda a violência para relatar a cada novo serviço ou organização de apoio que desestimula as mulheres a denunciar e procurar esses serviços. Assim, todos os profissionais envolvidos na assistência a mulher inserida no contexto de violência, conhecendo a relação e os efeitos negativos da violência na saúde, devem estar habilitados a identificar, desde o início, os casos de violência e, conhecendo previamente os instrumentos sociais de apoio, direcionar a usuária ao longo desta rede intersetorial (NETTO LA, et al., 2017; AIRBOT J, et al., 2018; BEARZI PSS, et al. 2020)

Portanto, a criação de um protocolo, a ser aperfeiçoado a partir das experiências e evidências que forem surgindo, com o escopo de orientar os profissionais de saúde, sobretudo os da APS, a despeito da forma de acolher e realizar a escuta qualificada, do necessário interrogatório sobre a violência e os possíveis direcionamentos, bem como fornecer um roteiro de questionamentos a ser realizado pelo Agente Comunitário de Saúde para que sejam capazes de identificar precocemente os casos de violência (D'OLIVEIRA AFPL, et al. 2020; SILVA VG e RIBEIRO PM, 2020).

Não menos importante é batalhar pelo fim do modelo biomédico em detrimento da implantação do modelo biopsicossocial, com ênfase na humanização do cuidado e todas as diretrizes que a Política Nacional de Humanização propõe. Necessário ainda o compromisso e responsabilização dos gestores para que possa haver uma reordenação e articulação da rede, partindo dos fluxos e sistemas internos, abarcando todos que integrem a rede intersetorial (AMARIJO CL, et al., 2020; ARBOIT J, et al., 2017). 


\section{CONSIDERAÇÕES FINAIS}

Evidenciou-se ausência de protocolos com diretrizes que direcionem as condutas dos profissionais de saúde, a fim de melhor prestar assistência às vítimas da violência contra a mulher, restando apenas ações isoladas e desarticuladas com outros órgãos e entidades que podem apoiar as mulheres neste momento de vulnerabilidade e priorizando os aspectos legais e medidas judiciais na solução dos problemas. De modo que este estudo, buscou contribuir para o reconhecimento da necessidade de criação de mecanismos, a serem aperfeiçoados a partir das experiências e evidências surgidas, que sistematize e oriente atuação profissional, a fim de otimizar as rotinas dos serviços de saúde no atendimento integral às mulheres.

\section{REFERÊNCIAS}

1. AMARIJO CL, et al. Assimilação teórica e prática da violência doméstica: profissionais de enfermagem atendendo vítimas na Atenção Primária. Revista de Enfermagem da UERJ, 2018; 26: e33874.

2. ARBOIT J, et al. Atenção à saúde de mulheres em situação de violência: desarticulação dos profissionais em rede. Revista da Escola de Enfermagem da USP, 2017; 51 : e03207.

3. ARBOIT J, et al. Violência doméstica contra mulheres rurais: práticas de cuidado desenvolvidas por agentes comunitários. Saúde e Sociedade, 2018; 27(2): 506-517.

4. BEARZI PSS, et al. Trilhas para o enfrentamento da violência contra a mulher. Revista de Estudos Feministas, 2020; 28(3): e60162.

5. BHONA FM, et al. Socioeconomic Factors and Intimate Partner Violence: a household survey. Temas em Psicologia, 2019; 27(1): 205-2018.

6. CAMPOS 10 , et al. Mulheres em situações de violência doméstica acompanhadas em um Centro de Atenção Psicossocial. Revista NUFEN, 2020; 12(3): 116-138.

7. D'OLIVEIRA AFPL, et. al. Obstáculos e facilitadores para o cuidado de mulheres em situação de violência doméstica na Atenção Primária em Saúde: uma revisão sistemática. Interface - comunicação, saúde, educação, 2020; 24: e190164.

8. ESPERANDIO EG, et al. Violência íntima: experiência de mulheres na Atenção Primária à Saúde no Rio de Janeiro, RJ, Brasil. Inteface - comunicação, saúde, educação, 2020; 24(1): e190707.

9. MACHADO MES, et al. Percepção dos profissionais de saúde sobre violência contra a mulher: estudo descritivo. Online Braz J Nurs, 2017;16(1): 209-217.

10. MAGALHÃES VMPR, et al. Validação de álbum seriado para enfermeiros da Atenção Básica sobre violência doméstica contra a mulher. Cogitarem enfermagem, 2020; 24: e62729.

11. MENDES KDS, et al. Revisão Integrativa: método de pesquisa para a incorporação de eidências na saúde e na enfermagem. Texto Contexto - Enfermagem, 2008; 17(4): 758-764.

12. MENDONÇA CF, et al. Violência na Atenção Primária em Saúde no Brasil: uma revisão integrativa de literatura. Ciência \& Saúde Coletiva, 2020; 25(6): e16977.

13. MOTA JA, AGUIAR RS. Percepções de enfermeiros da Atenção Primária noo atendimento às mulheres vítimas de violência. Revista Nursing, 2020; 23(262): 3648-3651.

14. NASCIMENTO VF, et al. Desafios no atendimento aos casos de violência doméstica contra a mulher em um município matogrossense. Arquivos de Ciências da Saúde UNIPAR, 2019; 23(1): 15-22.

15. NETTO LA, et al. As redes de apoio às mulheres em situação de violência pelo parceiro íntimo. Texto Contexto Enfermagem, 2017; 26(2): e07120015

16. OLIVEIRA GL, et al. Domestic violence against women according to the viewpoint of family health strategy teams. Revista de Pesquisa Cuidado é Fundamental Online, 2020; 12: 850-855.

17. POMPEO DA, et. al. Revisão integrativa: etapa inicial do processo de validação de diagnóstico de enfermagem. Acta Paulista de Enfermagem, 2009; 22(4): 434-438.

18. SACRAMENTO LT, REZENDE MM. Violências: lembrando alguns conceitos. Aletheia, 2006; 24: 95-104.

19. SANTOS, SC, et al. Violência contra a mulher: como os profissionais na Atenção Primária à Saúde estão enfrentando esta realidade?, Revista Saúde e Pesquisa, 2018; 11(2): 359-368.

20. SEHNEM GD, et al. Violência contra as mulheres: atuação da enfermeira na Atenção Primária à Saúde. Revista de Enfermagem da UFSM, 2019; 9(62): 1-19.

21. SIGNORELLI MC, et al. Domestic violence against womem, public policies and community health workers in Brazilian Primary Health Care. Ciência \& Saúde Coletiva, 2018; 23(1): 93-102.

22. SILVA NNF, et al. Atuação dos enfermeiros da Atenção Básica a mulheres em situação de violência. Enfermagem em Foco, 2017; 8(3): 7074.

23. SILVA VG, RIBEIRO PM. Violência contra as mulheres na prática de enfermeiras da Atenção Primária à Saúde. Escola Anna Nery, 2020; 24(4): e20190371. 